\title{
Statistical Entropy of Quantum Black Holes in the Presence of Quantum Gravity Effects
}

\author{
A. D. Kamali $(\mathbb{D})$ and H. Shababi $i$ \\ Young Researchers and Elites Club, Science and Research Branch, Islamic Azad University, Tehran, Iran \\ Correspondence should be addressed to A. D. Kamali; adkamali@srbiau.ac.ir
}

Received 29 January 2018; Revised 30 March 2018; Accepted 3 April 2018; Published 26 June 2018

Academic Editor: Elias C. Vagenas

Copyright (c) 2018 A. D. Kamali and H. Shababi. This is an open access article distributed under the Creative Commons Attribution License, which permits unrestricted use, distribution, and reproduction in any medium, provided the original work is properly cited. The publication of this article was funded by $\mathrm{SCOAP}^{3}$.

\begin{abstract}
We investigate the effects of deformed algebra, admitted from minimal length, on canonical description of quantum black holes. Using the modified partition function in the presence of all orders of the Planck length, we calculate the thermodynamical properties of quantum black holes. Moreover, after obtaining some thermodynamical quantities including internal energy, entropy, and heat capacity, we conclude that, at high temperature limits due to the decreasing of the number of microstates, the entropy tends to upper bounds.
\end{abstract}

\section{Introduction}

As it is known, one of the most important results of introducing General Relativity (GR) is the development of human's insight of the universe. However, at high energy levels or very short distances, the study of the attributes of physical phenomena leads to new problems which makes an important contribution to our understanding of the nature. These issues are revealed specially in Planck scales where quantum effects play essential roles just like the gravitational effects.

Although GR seems to be a purely classical theory, for some major applications such as cosmology and black hole $(\mathrm{BH})$ theories, the quantization of gravity is the main problem of theoretical physics community. Since there is not a full theory of quantum gravity (QG), different approaches are introduced towards QG from phenomenological grounds. For instance, the canonical quantum theory of gravity which was first introduced by De Witt can be considered in this category [1]. It is worth mentioning that all approaches to QG scenario including string theory, noncommutative geometry, and loop quantum gravity (LQG) have shown the existence of a minimal measurable length $[2,3]$.

One way to survey some phenomenological aspects of effective QG candidates is the deformation of algebraic structure of ordinary quantum mechanics. In this sense, the generalized uncertainty principle $[4,5]$ and noncommutative geometry $[6,7]$ can be mentioned as the most famous deformations which impose the ultraviolet and infrared cutoffs for the physical systems $[8,9]$. As it is known, noncommutative geometry imposes the ultraviolet (UV) and infrared (IR) cutoffs on the spacetime manifold $[8,9]$. According to the fact that the existence of UV and IR cutoffs is necessary for regularization and renormalization of quantum fields in curved spacetimes, the noncommutative geometry can be considered as an appropriate framework to formulate theories which deal with UV and IR cutoffs. It has been proved that quantum field theories in a noncommutative background are naturally regularized.

The study of black hole thermodynamics in quantum field theory which is governed in curved spacetime is interesting because it leads to black hole thermal emission via Hawking process [10-12]. Also, in order to calculate the standard statistical mechanics quantities of black holes, the Hamiltonian formulation of black hole is needed. It is worth mentioning that although formulating BH's Hamiltonian in the classical framework of GR is impossible, for some particular cases like the Schwarzschild BH, it is possible [13-15]. Quantization of the Schwarzschild mass is found out within this process which coincides with Bekenstein proposal $[16,17]$. It should be noted that the applications of quantum gravity have been widely studied. In particular, the quantum gravity can modify 
the thermodynamics of black hole, such as SchwarzschildTangherlini black hole $[18,19]$ and Reissner Nordstrom de Sitter quintessence black hole [20, 21], and the strength of the quantum gravitational effect can be constrained by the gravitational wave events [22].

In this paper, using the higher order deformed commutation relation $[X, P]=i \hbar \exp \left(\lambda P^{2}\right)$, we investigate semiclassical statistical mechanics which implies the existence of the minimal measurable length. Also, it is shown that our results coincide with those obtained from full quantum considerations in the limit of high temperature. Since this way is appropriate to investigate the thermodynamical properties of quantum black holes, we use that to study thermodynamics of the quantum black hole in new framework and find corrections to the Hawking entropy. It should be noted that our method can be very interesting because we obtain solutions without solving the Hamiltonian eigenvalue problem.

\section{Quantum Corrections to Black Holes}

It is known that when a pair of appropriate canonical coordinates $\left(\mathbf{m}, p_{m}\right)$ is identified, Schwarzschild black hole can be described as a canonical system which its Hamiltonian is described by its mass; that is, $H=\mathbf{m}$ [13-15]. But a canonical transformation takes place in a new canonical pair $\left(a, p_{a}\right)$, which leads to the following new Hamiltonian [13-15]:

$$
\begin{aligned}
H & =\frac{p_{a}^{2}}{2 a}+\frac{a}{2}, \\
\left|p_{m}\right| & =\int_{a}^{2 \mathbf{m}} \frac{d b}{\sqrt{2 \mathbf{m} b^{-1}-1}} \\
& =\sqrt{2 \mathbf{m} a-a^{2}}+\left(1-\frac{a}{\mathbf{m}}\right)+\frac{1}{2} \pi \mathbf{m} \\
p_{a} & =\operatorname{sgn}\left(p_{m}\right) \sqrt{2 \mathbf{m} a-a^{2}} .
\end{aligned}
$$

The ranges of the variables are $a>0$ and $-\infty<p_{a}<\infty$. The transformation is well-defined, one-to-one, and canonical. Using (1), the Wheeler-Dewitt equation is obtained as [2325]

$$
\frac{h^{2} G^{2}}{c^{6}} a^{-s-1} \frac{d}{d a}\left(a^{s} \frac{d}{d a} \psi(a)\right)=\left(a-\frac{2 G M}{c^{2}}\right) \psi(a) .
$$

For the special case, if we set $s=2$ (Indeed, there are various possibilities for ordering. Since the factor-ordering parameter will not affect the semiclassical calculations in minisuperspace models, one usually chooses a special value for it in a given model.), $R_{s}=2 G M / c^{2}, \psi(a)=(1 / a) U(a)$, and $\xi=a-R_{s}$, the corresponding Wheeler-DeWitt equation can be expressed in the form of a Schrödinger equation for a quantum harmonic oscillator as [23-26]

$$
\left(-\frac{1}{2} l_{p}^{2} E_{p} \frac{d^{2}}{d x^{2}}+\frac{E_{p}}{2 l_{p}^{2} E_{p}^{2}}\right) U(x)=\frac{R_{s}}{4 l_{p}} E_{s} U(x),
$$

where $l_{p}$ is the Planck length, $E_{p}=\sqrt{\hbar c^{2} / G}$ is the Planck energy, $E_{s}=M c^{2}$ is the black hole ADM energy, and
$R_{s}=2 G M / c^{2}$ is the Schwarzschild radius (where the variable $\xi$ indicates the gravitational degrees of freedom of the Schwarzschild black hole, defining the appropriate constants and considering the fact that the energy of excitations associated with variable $a$ is not positive as a phase coordinate. $\psi(a)=(1 / a) U(a)$ is the $\mathrm{BH}$ wave function. $)$.

After initial results which were given by Duff [27], the most important quantum correction to the Newtonian potential (NP) is derived by Donoghue, with the acceptance of GR as the basic theory of gravity. Also, Donoghue [28, 29] formulated GR as an effective field theory (EFT) and he established that interaction between two bodies can be considered as a potential gravitational energy [30-33]; that is,

$$
U(r)=-\frac{G M m}{r}\left(1+\frac{3 G(M+m)}{r c^{2}}+\frac{41}{10 \pi} \frac{\ell_{P}^{2}}{r^{2}}\right) .
$$

Clearly, the first term of correction has no power of $\hbar$. So, it is a classical effect and this is due to the nonlinear nature of GR. The second term of correction is a true quantum effect, linear in $\hbar$. Now, the potential which is generated by mass $M$ reads

$$
V(r)=-\frac{G M}{r}\left(1+\frac{3 G M}{r}\left(1+\frac{m}{M}\right)+\frac{41}{10 \pi} \frac{\ell_{P}^{2}}{r^{2}}\right) .
$$

Next, we attend to the effective potential which is obtained from a metric in general form

$$
d s^{2}=f(r) d t^{2}-g_{i k}\left(x_{1}, x_{2}, x_{3}\right) d x^{i} d x^{k}
$$

where $r=|\mathbf{x}|=\left(x_{1}^{2}+x_{2}^{2}+x_{3}^{2}\right)^{1 / 2}$ and $x_{1}, x_{2}$, and $x_{3}$ are the standard Cartesian coordinates. From (7), the standard form of the Schwarzschild metric, namely,

$$
d s^{2}=\left(1-\frac{2 G M}{r}\right) d t^{2}-\left(1-\frac{2 G M}{r}\right)^{-1} d r^{2}-r^{2} d \Omega^{2},
$$

in harmonic coordinates leads to

$$
\begin{aligned}
d s^{2}= & \left(\frac{R-G M}{R+G M}\right) d t^{2}-\left(\frac{R+G M}{R-G M}\right) d R^{2} \\
& -(R+G M)^{2} d \Omega^{2}
\end{aligned}
$$

where $R=r-G M$. For any general form of metric, we have [34]

$$
d s^{2}=f(r) d t^{2}-f(r)^{-1} d r^{2}-C(r) d \Omega^{2} .
$$

Now, form (7) and (10), we get

$$
\begin{aligned}
d s^{2}= & f(r) d t^{2}-\left(f(r)^{-1}-\frac{C(r)}{r^{2}}\right) \frac{1}{r^{2}}(\mathbf{x} \cdot d \mathbf{x})^{2} \\
& -\frac{C(r)}{r^{2}} d \mathbf{x}^{2} .
\end{aligned}
$$

The point that should be noted here is that for a particle far from the source which moves slowly in a stationary and weak gravitational field, that is, $r \rightarrow \infty$, the effective 
Newtonian potential is given by the metric (7). So, the effective Newtonian potential is

$$
V(r) \simeq \frac{1}{2}(f(r)-1) .
$$

According to [23-26], using the path integral method which is applied for the harmonic oscillator, quantum effects on thermodynamics of the Schwarzschild $\mathrm{BH}$ can be introduced. To this end, the modified harmonic potential and the quantum corrected Hamiltonian for Schwarzschild black holes read

$$
\begin{aligned}
V(x) & =\frac{m \omega^{2}}{2}\left(x^{2}+\frac{\beta_{\mathrm{Q}} \hbar^{2}}{12 m}\right), \\
H_{\mathrm{Q}} & =\frac{p^{2}}{2 m_{p}}+\frac{m_{p} \omega^{2} x^{2}}{2}+\frac{\beta_{\mathrm{Q}} E_{p}^{2}}{16 \pi},
\end{aligned}
$$

where $\omega$ is the frequency of the quantum harmonic oscillator, namely, $\omega=\sqrt{3 / 2 \pi}\left(E_{p} / \hbar\right)$, and $\beta_{Q}=\beta_{H}\left[1-1 / \beta_{H} M c^{2}+\right.$ $\left.\mathcal{O}\left(E_{p} / M c^{2}\right)\right]$ is the quantum corrected inverse $\mathrm{BH}$ temperature. As it is known, in thermodynamics for a quantum system, the partition function is given by

$$
Z=\frac{1}{h} \int e^{-\beta H(p, x)} d p d x,
$$

where $\beta=1 / T$ and $T$ is thermodynamical temperature of a system. Note that when there is not any deformed coordinates and momenta in the system, $x_{i}$ and $p_{i}$ are still ordinary canonically conjugate; that is, $\left\{x_{i}, x_{j}\right\}=\left\{p_{i}, p_{j}\right\}=0$ and $\left\{x_{i}, p_{j}\right\}=\delta_{i j}$. So, the resulted partition function gives

$$
\begin{aligned}
Z & =\frac{1}{h} \int_{-\infty}^{\infty} \int_{-\infty}^{\infty} e^{-\beta\left(p^{2} / 2 m_{p}+m_{p} \omega^{2} x^{2} / 2+\beta_{Q} E_{p}^{2} / 16 \pi\right)} d p d x \\
& =\frac{2 \pi T e^{-E_{p}^{2} / 16 \pi T^{2}}}{h \omega} .
\end{aligned}
$$

Next, we obtain Helmholtz free energy, entropy, internal energy, and specific heat capacity, respectively, as follows:

$$
\begin{aligned}
& F=-T \ln (Z)=-T \ln \left(\frac{2 \pi T}{h \omega}\right)+\frac{E_{p}^{2}}{16 \pi T}, \\
& S=\frac{\partial F}{\partial T}=\ln \left(\frac{2 \pi T}{h \omega}\right)+\frac{E_{p}^{2}}{16 \pi T^{2}}+1, \\
& U=-T^{2} \frac{\partial(F / T)}{\partial T}=\frac{E_{p}^{2}}{8 \pi T}+T, \\
& C=\frac{\partial U}{\partial T}=1-\frac{E_{p}^{2}}{8 \pi T^{2}} .
\end{aligned}
$$

Clearly, by setting $E_{p}=0$, we recover the classical quantities. In brick wall model [35-40], the ordinary uncertainty relation is given by

$$
\Delta x \Delta p \geq \frac{\hbar}{2}
$$

Then, the entropy of black hole can be obtained as follows [35-40]:

$$
\begin{aligned}
& S_{0}=\left.\beta^{2} \frac{\partial F_{0}}{\partial \beta}\right|_{\beta=\beta_{H}}=\frac{\beta^{2}}{\pi} \int_{r_{+}+\epsilon}^{L} d r \\
&\left.\cdot \frac{1}{\sqrt{f}} \int_{m \sqrt{f}}^{\infty} d \omega \frac{\omega e^{\beta \omega}\left(\omega^{2} / f-m^{2}\right)^{1 / 2}}{\left(e^{\beta \omega}-1\right)^{2}}\right|_{\beta=\beta_{H}},
\end{aligned}
$$

where $F_{0}$ is the Helmholtz free energy, $\epsilon$ is the ultraviolet regulator, $L$ is the infrared regulator, $\omega$ is the energy of particle, and $\beta$ is the inverse Hawking temperature. In massless case, the black hole entropy can be calculated as follows [35-39]:

$$
S_{0} \approx \frac{1}{12} \ln \left(\frac{1}{2 \Lambda \epsilon}\right),
$$

where $\Lambda$ is the cosmological constant. For the external case, we can assume that $\beta \rightarrow \infty$. So, we have

$$
S_{0}^{\text {ext }}=\left.\beta^{2} \frac{\partial F_{0}}{\partial \beta}\right|_{\beta \rightarrow \infty}=0 .
$$

Although these results include a logarithmic divergence, they will be changed due to the effects of GUP.

\section{The Effects of Deformed Algebra on Some Thermodynamical Quantities}

In quantum mechanical levels, the nature of UV limitation of Feynman propagator can be obtained by a nonlinear relation between the high energy momentum, $p$, and the wave vector of a particle, $f(k)$; that is, $p=f(k)$ [41-45].

Now, considering the situations in our paper, the standard momentum measure $d p$ should be changed to $d p \prod_{i}\left(\partial k_{i} / \partial p_{j}\right)$. From now on, we turn our attention to the identical case and work with one space-like dimension. Following [6-9, 41-45], the following is obtained:

$$
\frac{\partial p}{\partial k}=\hbar \exp \left(\alpha^{2} l_{p}^{2} p^{2}\right)
$$

where $\alpha$ is a dimensionless constant. In this step, using (21) the dispersion relation is given by

$$
k(p)=\frac{\sqrt{\pi}}{2 \alpha l_{p}} \operatorname{Erf}\left(\alpha l_{p} p\right)
$$

and the results can be obtained from the following representation in momentum space:

$$
X=i \hbar \exp \left(\lambda P^{2}\right) \partial_{p} \quad P=p,
$$

in which $\lambda=\alpha^{2} l_{p}^{2}$. The corrections to the standard Heisenberg algebra become effective in the so-called quantum regime, where the momentum and length scales are of the order of the Planck mass, $m_{p}$, and the Planck length, $l_{p}$, respectively. 
The algebra which is defined by (23) leads to the following generalized commutator:

$$
[X, P]=i \hbar \exp \left(\lambda P^{2}\right)
$$

and implies the generalized uncertainty principle, namely, GUP*,

$$
\Delta X \Delta P \geq \frac{\hbar}{2}\left\langle\exp \left(\lambda P^{2}\right)\right\rangle
$$

Now, if we use the property $(\Delta P)^{2}=\left\langle P^{2}\right\rangle-\langle P\rangle^{2}$, then the saturate $\mathrm{GUP}^{*}$ is obtained as

$$
(\Delta X)(\Delta P)=\frac{\hbar}{2} \exp \left(\lambda\left((\Delta P)^{2}+\langle P\rangle^{2}\right)\right) .
$$

Next, taking the square of this relation implies that

$$
u=W(u) e^{W(u)}
$$

where $W(u)=-2 \lambda(\Delta P)^{2}$ and $u=-\lambda \hbar^{2} / 2(\Delta X)^{2}$.

As is known, (27) is exactly indicated in the definition of the Lambert function [46] and its various branches are labeled by the integer $k=0, \pm 1, \pm 2, \ldots$. Now, it should be noted that if $u$ is a real number, (27) leads to a pair of real solutions for $-1 / e \leq u \leq 0$ which are denoted by $W_{-1}(u)$ and $W_{0}(u)$, respectively. Also, it can only have one real solution for $u \geq 0$, namely, $W_{0}(u)$, and for $-\infty<u<-1 / e$, there is no real solution.

Now, using (27) the uncertainty in momentum is expressed as

$$
\Delta P=\frac{\hbar}{2 \Delta X} e^{-(1 / 2) W\left(-\lambda \hbar^{2} / 2(\Delta X)^{2}\right)} .
$$

Then, according to the argument of the Lambert function (28) we have the condition $\lambda \hbar^{2} / 2(\Delta X)^{2} \leq 1 / e$ which leads to the following minimal uncertainty in position:

$$
(\Delta X)_{\min }=\hbar \sqrt{\frac{e \lambda}{2}}
$$

Here, the momentum uncertainty can be obtained in terms of the minimal length as

$$
\Delta P=\frac{\hbar}{2 \Delta X} \exp \left(-\frac{1}{2} W\left(-\frac{1}{e}\left(\frac{\Delta X_{\min }}{\Delta X}\right)^{2}\right)\right) .
$$

From now on, for simplicity we set $l_{p}=m_{p}^{-1}=T_{p}^{-1}=\sqrt{G}=1$.

Now, using the deformed algebra (24), the canonical partition function for a system reads

$$
\begin{aligned}
Z_{\text {new }} & =\frac{1}{h} \int_{\Gamma} \omega \exp (-\beta H) \\
& =\frac{1}{h} \int d q \int \frac{d p}{J(q, p)} \exp (-\beta H(q, p)),
\end{aligned}
$$

where $H(X, P)$ is the Hamiltonian of the system, $T$ is temperature, and $J=\partial\left(X_{1}, P_{1}, \ldots, X_{D}, P_{D}\right) / \partial\left(x_{1}, p_{1}, \ldots, x_{D}, p_{D}\right)$ is the Jacobian of the transformation in $D$-dimensions. Since the Jacobian can be read off from the deformed Poisson brackets, in one dimension, it is concluded that [47]

$$
J=\frac{\partial\left(X_{1}, P_{1}\right)}{\partial\left(x_{1}, p_{1}\right)}=\left\{X_{1}, P_{1}\right\} \text {. }
$$

So, we obtain

$$
J=\exp \left(\lambda P^{2}\right)
$$

Consequently, using the new partition function, it is easy to investigate the effects of deformed phase space on thermodynamical properties of the statistical systems.

\section{Deformed Algebra on the Quantum Black Hole}

Based on Section 2, the modified Hamiltonian of the Schwarzschild black holes is given by (13). Now, using the deformed algebra (24), the new partition function is obtained as

$$
\begin{aligned}
Z & =\frac{1}{h} \iint \frac{e^{-\beta H_{Q}(X, P)}}{J(X, P)} d X d P \\
& =\frac{1}{h} \iint_{-\infty}^{+\infty} \frac{e^{-\beta\left(P^{2} / 2 m+m \omega^{2} x^{2} / 2+\beta E_{p}{ }^{2} / 16 \pi\right)}}{e^{\lambda P^{2}}} d X d P,
\end{aligned}
$$

which concludes that

$$
Z_{\mathrm{GUP}}(T, \lambda)=\frac{2 \pi e^{-E_{p}^{2} / 16 \pi T^{2}}}{h \omega} \sqrt{\frac{T^{2}}{1+2 m \lambda T}} .
$$

Next, we apply the modified partition function (35) to obtain some thermodynamical quantities such as Helmholtz free energy, entropy, internal energy, and heat capacity, respectively, as follows:

$$
\begin{aligned}
F= & -T \ln \left(\frac{2 \pi \sqrt{T^{2} /(1+2 m \lambda T)}}{h \omega}\right)+\frac{E_{p}^{2}}{16 \pi T}, \\
S= & \left(\frac{1}{2}+\frac{E_{p}^{2}}{16 \pi T^{2}}+\frac{1}{2+4 m \lambda T}\right) \\
& +\ln \left(\frac{2 \pi \sqrt{T^{2} /(1+2 m \lambda T)}}{h \omega}\right), \\
U= & \frac{E_{p}^{2}}{8 \pi T}+\left(1+\frac{1}{1+2 m \lambda T}\right) \frac{T}{2} \\
C= & \frac{1}{2}-\frac{E_{p}^{2}}{8 \pi T^{2}}+\frac{1}{2(1+2 m \lambda T)^{2}} .
\end{aligned}
$$

In this step, because of the duality properties of position and momentum operators, we assume that $\Delta X_{\min } \propto \Delta P_{\max }$. Now, saturating the inequality in relation (28), we obtain

$$
\begin{aligned}
& \Delta X=\Delta X_{\min } \longrightarrow \\
& \Delta P=\Delta P_{\max }=\frac{1}{\sqrt{2 \lambda}} .
\end{aligned}
$$




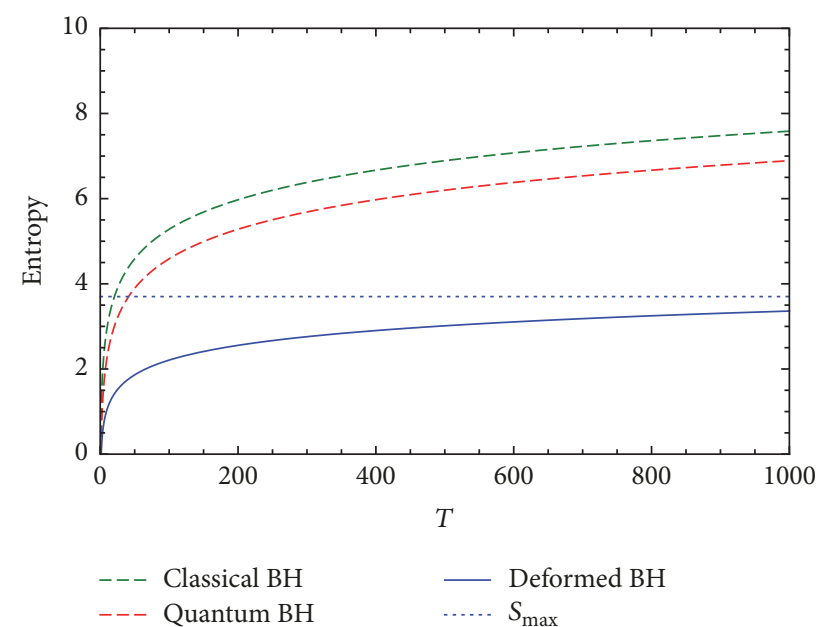

FIGURE 1: Entropy of quantum black hole versus temperature for $m=h=E_{p}=\lambda=1$. As we have obtained this result in [2325], in high energy limits and near the Plank length scale, the effects of quantum gravity can be considered as a perturbation sentence in quantum mechanical theory which leads to reducing the photon radiation from the distant observer's point of view. So, black holes in quantum gravity frameworks have numerically smaller entropy than the nondeformed black holes in four dimensions.

Thus, using the maximal momentum (37), the partition function is expressed as

$$
\begin{aligned}
& Z=\frac{1}{h} \iint_{-P_{\max }}^{+P_{\max }} \frac{e^{-\beta H_{Q}(X, P)}}{e^{\lambda P^{2}}} d X d P \\
& =\frac{e^{-\beta^{2} E_{p}^{2} / 16 \pi}}{h \omega} \sqrt{\frac{1}{\beta(\beta+2 m \lambda)}} \operatorname{Erf}\left(\sqrt{\frac{\beta+2 m \lambda}{4 m \lambda}}\right),
\end{aligned}
$$

which concludes the following entropy:

$$
\begin{aligned}
S & =\frac{1}{2}+\frac{E_{p}^{2}}{16 \pi T^{2}}+\frac{1}{2+4 m T \lambda} \\
& -\frac{e^{-(1+m \lambda T) / 4 m \lambda T} \sqrt{(1+2 m \lambda T) / m \lambda T}}{2 \sqrt{\pi}(1+2 m \lambda T) \operatorname{erf}(\sqrt{(1+2 m \lambda T) / 4 m \lambda T})} \\
& +\ln \left[\frac{\pi \sqrt{T^{2} /(1+2 m \lambda T)} \operatorname{erf}(\sqrt{(1+2 m \lambda T) / 4 m \lambda T})}{2 h \omega}\right] .
\end{aligned}
$$

To investigate the results more clear, we have depicted entropy of quantum black hole versus temperature in Figure 1, in which it is shown that the modified entropy increases with a slower slope (the blue solid line) more than the nondeformed one (the red dashed line). Moreover, the entropy approaches a maximum bound (the blue dotted line) at very high temperature limit in deformed algebra which is not observed in standard framework.
The phase space volume in the $(1+1)$ dimension is changed from $2 \pi$ to $2 \pi e^{\lambda p^{2}}$ and the number of quantum state with energy less than $\epsilon$ is obtained as [35-40]

$$
\begin{aligned}
n_{0}(\omega) & =\frac{1}{2 \pi} \int d r d p_{r} \\
& =\frac{1}{\pi} \int_{r_{+}+\epsilon}^{L} d r \frac{1}{\sqrt{f}}\left(\frac{\omega^{2}}{f}-m^{2}\right)^{1 / 2},
\end{aligned}
$$

where $\omega$ is a parameter of Klein-Gordon equation and $m$ in the mass of the scalar field in brick wall model.

Equation (40) can be related to

$$
\begin{aligned}
n_{I}(\omega) & =\frac{1}{2 \pi} \int d r d p_{r} e^{-\lambda p^{2}} \\
& =\frac{1}{2 \pi} \int d r \frac{1}{\sqrt{f}} \frac{\left(\omega^{2} / f-m^{2}\right)^{1 / 2}}{e^{\lambda\left(\omega^{2} / f-m^{2}\right)}} .
\end{aligned}
$$

Now, using (41), the free energy can be derived as [35-40]

$$
F_{0}=-\frac{1}{\pi} \int_{r_{+}+\epsilon}^{L} d r \frac{1}{\sqrt{f}} \int_{m \sqrt{f}}^{\infty} d \omega \frac{\left(\omega^{2} / f-m^{2}\right)^{1 / 2}}{e^{\beta \omega}-1}
$$

which turns to be a new equation as follows:

$$
\begin{aligned}
F_{I} & =-\int_{m \sqrt{f}}^{\infty} d \omega \frac{n_{I}(\omega)}{e^{\beta \omega}-1} \\
& =-\frac{1}{\pi} \int d r \frac{1}{\sqrt{f}} \int_{m \sqrt{f}}^{\infty} d \omega \frac{\left(\omega^{2} / f-m^{2}\right)^{1 / 2}}{\left(e^{\beta \omega}-1\right) e^{\lambda\left(\omega^{2} / f-m^{2}\right)}} .
\end{aligned}
$$

From (43), the entropy of $\mathrm{BH}$ near the event horizon, that is, in the range of $\left(r_{+}, r_{+}+\epsilon\right)$ and $f \rightarrow 0$, is

$$
S_{0}=\left.\frac{\beta^{2}}{\pi} \int_{r_{+}+\epsilon}^{L} d r \frac{1}{\sqrt{f}} \int_{m \sqrt{f}}^{\infty} d \omega \frac{\omega e^{\beta \omega}\left(\omega^{2} / f-m^{2}\right)^{1 / 2}}{\left(e^{\beta \omega}-1\right)^{2}}\right|_{\beta=\beta_{H}} .
$$

In deformed algebra, (44) changes to

$$
\begin{aligned}
S_{I} & =\frac{\beta^{2}}{\pi} \int d r \frac{1}{\sqrt{f}} \int_{m \sqrt{f}}^{\infty} \frac{\omega\left(\omega^{2} / f-m^{2}\right)^{1 / 2} e^{\beta \omega}}{e^{2 \beta \omega-2} e^{\lambda\left(\omega^{2} / f-m^{2}\right)}} d \omega \\
& =\frac{1}{\pi} \int_{r_{+}}^{r_{+}+\varepsilon} d r \frac{1}{\sqrt{f}} \int_{0}^{\infty} \frac{f^{-1 / 2} \beta^{-1} x^{2}}{\left(1-e^{-x}\right)\left(e^{x}-1\right) e^{\lambda x^{2} / \beta^{2} f}} d x,
\end{aligned}
$$

where $x=\beta \omega$. It should be noted that as $f \rightarrow 0, \omega^{2} / f$ is the dominant term in $\omega^{2} / f-m^{2}$. The thermodynamic properties near the horizon $r_{+}, r_{+}+\epsilon$ are related to a proper distance of the order of the minimal length; that is, $\Delta x \approx \lambda$ [35-40]. So, we have

$$
\lambda=\int_{r_{+}}^{r_{+}+\epsilon} \frac{d r}{\sqrt{f(r)}}
$$


where $\lambda$ is considered as a lower bound. Then, we obtain entropy as

$$
S_{I}=\frac{1}{\pi \lambda} \int_{r_{+}}^{r_{+}+\varepsilon} \frac{d r}{\sqrt{f(r)}} \int_{0}^{\infty} d X \frac{a^{2} X^{2}}{\left(e^{a X / 2}-e^{-a X / 2}\right)^{2} e^{X^{2}}},
$$

where

$$
x=\beta \sqrt{\frac{f}{\lambda}} X=a X
$$

Thus

$$
S_{I}=\frac{1}{\pi} \Sigma_{I}=\frac{1}{\pi} \int_{0}^{\infty} \frac{a^{2} X^{2}}{\left(e^{a X / 2}-e^{-a X / 2}\right)^{2} e^{X^{2}}} d X .
$$

Obviously, if we set $r \rightarrow r_{+}, f \rightarrow 0$, and $a \rightarrow 0$, it is concluded that

$$
\lim _{a \rightarrow 0} \frac{a^{2} X^{2}}{\left(e^{a X / 2}-e^{-a X / 2}\right)^{2}}=1 .
$$

Therefore,

$$
\begin{aligned}
& \Sigma_{I}=\int_{0}^{\infty} \frac{d X}{e^{X^{2}}}=\frac{\sqrt{\pi}}{2}, \\
& S_{I}=\frac{1}{\pi} \Sigma_{I}=\frac{1}{2 \sqrt{\pi}} .
\end{aligned}
$$

So, we deduce that $S_{I}$ is finite and independent of any parameter. Also, we obtain that, in contrast to the brick wall method, there is no divergence due to the effect of the GUP on the quantum states [35-40].

We now want to consider this issue from the tunneling picture's point of view in $\mathrm{WKB}$ approximation. As it is shown in $[48,49]$, all of the tunneling probabilities of classical forbidden trajectory from inside to outside of the black hole horizon are given by

$$
\Gamma \sim e^{-2 \operatorname{ImI}}=e^{-E / T}=\frac{e^{S_{f}}}{e^{S_{i}}}=e^{\Delta S},
$$

where Im is the imaginary part and $\mathbf{I}$ is the classical action of trajectory. Also, $E$ is the energy of massless particle which is trajected from a BH in the form of a massless shell. Moreover, $\Delta S$ is the difference between final and initial values of the black hole entropy. The corrected entropy is given by [48-51]

$$
S_{\text {new }}=S_{\mathrm{BH}}+\zeta,
$$

where $\zeta$ is the extra terms of entropy in the quantum gravity framework. Thus, the corrected entropy is given by

$$
\Delta S_{\text {new }}=\Delta S_{\mathrm{BH}}+\Delta \zeta
$$

in which

$$
\begin{aligned}
\Delta S_{\mathrm{BH}} & =S_{\mathrm{BH}}(M-E)-S_{\mathrm{BH}}(M), \\
\Delta \zeta & =\zeta(M-E)-\zeta
\end{aligned}
$$

and $M$ is the mass of $\mathrm{BH}$. In usual tunneling radiation, the tunneling probability does not consider the bound. However, if the generalized second law is considered, it seems that the bound is also valid on the tunneling radiation rate. Substituting (54) into (52), the following is obtained:

$$
\Gamma_{\text {new }} \sim \Gamma_{\mathrm{BH}} e^{\Delta \zeta} .
$$

It is worthwhile to note that there is difference between qualitative behavior of the solutions of the exact equations for the tunneling probability, which is explained by the bound on the tunneling probability, and the photon emission [50-54].

This bound depends on the quantum gravity models. Existence of an exponential coefficient in the corrected tunneling probability in (56) predicts a generalized quantum tunneling through the horizon of the black hole, which obtains from the quantum gravitational effects on the black hole radiation $[50,51]$.

\section{Summary and Conclusions}

In this paper, using deformed algebra which was admitted from a minimal measurable length, we investigated quantum black holes in canonical ensemble. To this end, some thermodynamical quantities including partition function, Helmholtz free energy, entropy, internal energy, and specific heat capacity were obtained. For more investigations, we plotted entropy versus temperature which showed that the modified entropy of the quantum black hole increased with a slower slope more than the nondeformed ones. Next, we obtained, in contrast to the standard models at high temperature limits, that the entropy approached maximum bounds. Also, we concluded that, in contrast to the brick wall method, there was no divergence due to the effect of the GUP on the quantum states. It is worth mentioning that our results of existing a maximal bound in high temperature limits are fully compatible with those obtained in gravity's rainbow, modified dispersion relation, polymer quantum gravity, and noncommunicative setups. Note that the ordinary physical quantities are recovered when $\lambda \rightarrow 0$.

\section{Conflicts of Interest}

The authors declare that there are no conflicts of interest regarding the publication of this paper.

\section{References}

[1] B. S. De Witt, "Quantum theory of gravity. II. The manifestly covariant theory," Physical Review, vol. 160, p. 1113, 1967.

[2] L. Garay, "Quantum gravity and minimum length," International Journal of Modern Physics A, vol. 10, no. 2, pp. 145-165, 1995.

[3] A. Ashtekar and J. Lewandowski, "Background independent quantum gravity: a status report," Classical and Quantum Gravity, vol. 21, no. 15, pp. R53-R152, 2004.

[4] A. Kempf, G. Mangano, and R. B. Mann, "Hilbert space representation of the minimal length uncertainty relation," Physical Review D: Particles, Fields, Gravitation and Cosmology, vol. 52, no. 2, pp. 1108-1118, 1995. 
[5] S. Hossenfelder, "Minimal length scale scenarios for quantum gravity," Living Reviews in Relativity, vol. 16, no. 2, 2013.

[6] P. Nicolini, A. Smailagic, and E. Spallucci, "Noncommutative geometry inspired Schwarzschild black hole," Physics Letters B, vol. 632, no. 4, pp. 547-551, 2006.

[7] E. Spallucci, A. Smailagic, and P. Nicolini, "Trace anomaly on a quantum spacetime manifold," Physical Review D: Particles, Fields, Gravitation and Cosmology, vol. 73, no. 8, Article ID 084004, 11 pages, 2006.

[8] A. Kempf, "Quantum field theory with nonzero minimal uncertainties in positions and momenta," High Energy Physics, 1994, https://arxiv.org/abs/hep-th/9405067.

[9] A. Kempf and G. Mangano, "Minimal length uncertainty relation and ultraviolet regularization," Physical Review D, vol. 55, no. 12, pp. 7909-7920, 1997.

[10] S. W. Hawking, "Black hole explosions?" Nature, vol. 248, no. 5443, pp. 30-31, 1974.

[11] S. W. Hawking, "Particle creation by black holes," Communications in Mathematical Physics, vol. 43, no. 3, pp. 199-220, 1975.

[12] G. W. Gibbons and S. W. Hawking, "Action integrals and partition functions in quantum gravity," Physical Review D: Particles, Fields, Gravitation and Cosmology, vol. 15, no. 10, pp. 2752-2756, 1977.

[13] J. Mäkelä and P. Repo, "Quantum-mechanical model of the Reissner-Nordström black hole," Physical Review D: Particles, Fields, Gravitation and Cosmology, vol. 57, no. 8, pp. 4899-4916, 1998.

[14] J. Louko and S. N. Winters-Hilt, "Hamiltonian thermodynamics of the Reissner-Nordström-anti-de Sitter black hole," Physical Review D: Particles, Fields, Gravitation and Cosmology, vol. 54, no. 4, pp. 2647-2663, 1996.

[15] D. R. Brill, J. Louko, and P. Peldán, “Thermodynamics of (3+1)-dimensional black holes with toroidal or higher genus horizons," Physical Review D: Particles, Fields, Gravitation and Cosmology, vol. 56, no. 6, pp. 3600-3610, 1997.

[16] J. D. Bekenstein, "Black holes and entropy," Physical Review D: Particles, Fields, Gravitation and Cosmology, vol. 7, pp. 23332346, 1973.

[17] J. D. Bekenstein, "Generalized second law of thermodynamics in black-hole physics," Physical Review D: Particles, Fields, Gravitation and Cosmology, vol. 9, no. 12, pp. 3292-3300, 1974.

[18] Z. W. Feng, H. L. Li, X. T. Zu, and S. Z. Yang, "Quantum corrections to the thermodynamics of Schwarzschild-Tangherlini black hole and the generalized uncertainty principle," The European Physical Journal C, vol. 76, article 212, 2016.

[19] Y. S. Myung, "Stability of Schwarzschild black holes in fourthorder gravity revisited," Physical Review D: Particles, Fields, Gravitation and Cosmology, vol. 88, no. 2, 2013.

[20] Z. W. Feng, L. Zhang, and X. T. Zu, "The remnants in Reissner-Nordström-de Sitter quintessence black hole," Modern Physics Letters A, vol. 29, no. 26, Article ID 1450123, 2014.

[21] W.-B. Liu, "Reissner-Nordstrom black hole entropy inside and outside the brick wall," Chinese Physics Letters, vol. 20, no. 3, pp. 440-443, 2003.

[22] Z.-W. Feng, S.-Z. Yang, H.-L. Li, and X.-T. Zu, "Constraining the generalized uncertainty principle with the gravitational wave event GW150914," Physics Letters B, vol. 768, pp. 81-85, 2017.

[23] O. Obregón, M. Sabido, and V. I. Tkach, "Entropy using path integrals for quantum black hole models," General Relativity and Gravitation, vol. 33, no. 5, pp. 913-919, 2001.
[24] A. Bina, S. Jalalzadeh, and A. Moslehi, "Quantum black hole in the generalized uncertainty principle framework," Physical Review D: Particles, Fields, Gravitation and Cosmology, vol. 81, no. 2, Article ID 023528, 2010.

[25] R. P. Feynman and A. R. Hibbs, Quantum Mechanics and Path Integrals, McGrawHill, New York, NY, USA, 2010.

[26] P. Bargueño and E. C. Vagenas, "Semiclassical corrections to black hole entropy and the generalized uncertainty principle," Physics Letters B, vol. 742, pp. 15-18, 2015.

[27] M. J. Duff, "Quantum corrections to the Schwarzschild solution," Physical Review D: Particles, Fields, Gravitation and Cosmology, vol. 9, no. 6, pp. 1837-1839, 1974.

[28] J. F. Donoghue, "Leading quantum correction to the Newtonian potential," Physical Review Letters, vol. 72, no. 19, pp. 2996-2999, 1994.

[29] J. F. Donoghue, "General relativity as an effective field theory: the leading quantum corrections," Physical Review D: Particles, Fields, Gravitation and Cosmology, vol. 50, no. 6, pp. 3874-3888, 1994.

[30] G. G. Kirilin and I. B. Khriplovich, "Quantum power correction to the newton law," Journal of Experimental and Theoretical Physics, vol. 95, no. 6, pp. 981-986, 2002.

[31] N. E. J. Bjerrum-Bohr, J. F. Donoghue, and B. R. Holstein, "Quantum corrections to the Schwarzschild and Kerr metrics," Physical Review D: Particles, Fields, Gravitation and Cosmology, vol. 68, p. 084005, 2003, Erratum-ibid.D 71, 069904 (2005).

[32] N. E. Bjerrum-Bohr, J. F. Donoghue, and B. R. Holstein, "Quantum corrections to the Schwarzschild and Kerr metrics," Physical Review D: Particles, Fields, Gravitation and Cosmology, vol. 67, no. 6, p. 084033, 2003, rratum-ibid.D 71, 069903 (2005).

[33] A. Akhundov and A. Shiekh, "A review of leading quantum gravitational corrections to newtonian gravity," Electronic Journal of Theoretical Physics, vol. 17, no. 1, 2008.

[34] S. Weinberg, Gravitation \& Cosmology, John Wiley \& Sons, New York, NY, USA, 1972.

[35] G. 't Hooft, "On the quantum structure of a black hole," Nuclear Physics B, vol. 256, no. 4, pp. 727-745, 1985.

[36] W. Kim, Y.-W. Kim, and Y.-J. Park, "Entropy of a charged black hole in two dimensions without cutoff," Physical Review D: Particles, Fields, Gravitation and Cosmology, vol. 75, no. 12, Article ID 127501, 2007.

[37] Y.-W. Kim and Y.-J. Park, "Entropy of the Schwarzschild black hole to all orders in the Planck length," Physics Letters B, vol. 655, no. 3-4, pp. 172-177, 2007.

[38] M. Eune and W. Kim, "Lifshitz scalar, brick wall method, and generalized uncertainty principle in Hořava-Lifshitz gravity," Physical Review D: Particles, Fields, Gravitation and Cosmology, vol. 82, no. 12, Article ID 124048, 2010.

[39] F. H. Liu, Y. H. Chen, H. R. Wei, and B. C. Li, "Fermion's tunnelling with effects of quantum gravity," Advances in High Energy Physics, vol. 2013, Article ID 432412, 6 pages, 2013.

[40] A. N. Tawfik and E. A. El Dahab, "Corrections to entropy and thermodynamics of charged black hole using generalized uncertainty principle," International Journal of Modern Physics A, vol. 30, no. 9, 2015.

[41] S. Hossenfelder, "Interpretation of quantum field theories with a minimal length scale," Physical Review D: Particles, Fields, Gravitation and Cosmology, vol. 73, Article ID 105013, 2006.

[42] S. Hossenfelder, "A note on theories with a minimal length," Classical and Quantum Gravity, vol. 23, no. 5, pp. 1815-1821, 2006. 
[43] K. Nouicer and M. Debbabi, "Canonical quantization of nonanticommutative scalar field theory," Physics Letters A, vol. 361, no. 4-5, pp. 305-315, 2007.

[44] K. Nouicer, "Quantum-corrected black hole thermodynamics to all orders in the Planck length," Physics Letters B, vol. 646, no. 2-3, pp. 63-71, 2007.

[45] K. Nouicer, "Black hole thermodynamics to all orders in the Planck length in extra dimensions," Classical and Quantum Gravity, vol. 24, no. 23, pp. 5917-5934, 2007.

[46] R. M. Corless, G. H. Gonnet, D. E. G. Hare, and D. E. Knuth, "On the Lambert $W$ function," Advances in Computational Mathematics, vol. 5, no. 1, pp. 329-359, 1996.

[47] T. V. Fityo, "Statistical physics in deformed spaces with minimal length,” Physics Letters A, vol. 372, no. 37, pp. 5872-5877, 2008.

[48] P. Kraus and F. Wilczek, "Self-interaction correction to black hole radiance," Nuclear Physics B, vol. 433, no. 2, pp. 403-420, 1995.

[49] P. Kraus and F. Wilczek, "Effect of self-interaction on charged black hole radiance," Nuclear Physics B, vol. 437, no. 1, pp. 231242, 1995.

[50] H. Soltani, A. Damavandi Kamali, and K. Nozari, "Microblack holes thermodynamics in the presence of quantum gravity effects," Advances in High Energy Physics, vol. 2014, Article ID 247208, 12 pages, 2014.

[51] K. Nozari, S. Saghafi, and A. Damavandi Kamali, "Black hole thermodynamics with a modified dispersion relation admitting minimal length and maximal momentum," Astrophysics and Space Science, vol. 357, no. 2, 2015.

[52] R. J. Adler, P. Chen, and D. I. Santiago, "The generalized uncertainty principle and black hole remnants," General Relativity and Gravitation, vol. 33, no. 12, pp. 2101-2108, 2001.

[53] P. Chen and R. J. Adler, "Black hole remnants and dark matter," Nuclear Physics B, vol. 124, p. 103, 2003.

[54] M. H. P. M. van Putten, "Free fall onto evaporating black holes at the quantum limit," General Relativity and Quantum Cosmology, 2015, https://arxiv.org/abs/1506.08075. 

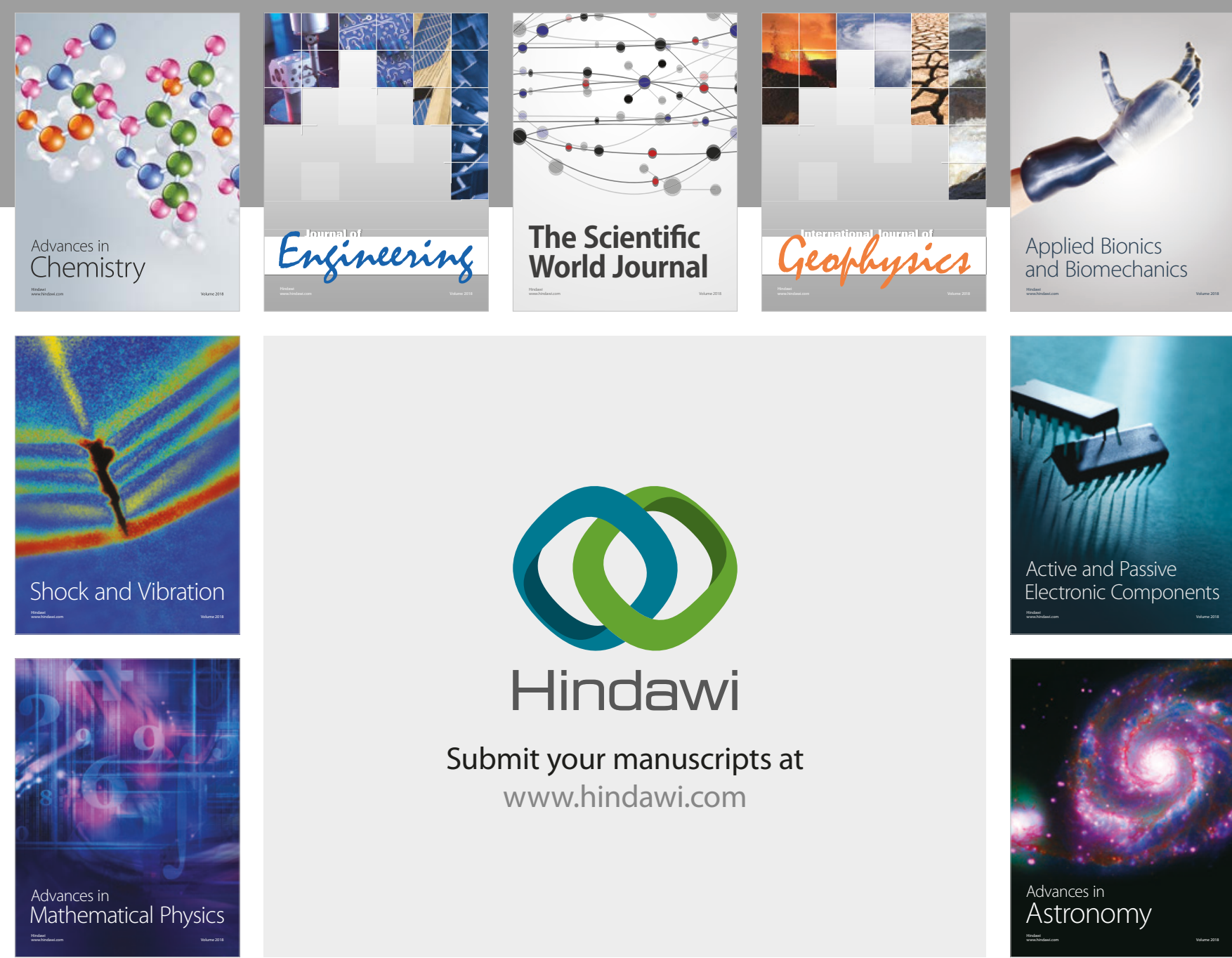

Submit your manuscripts at

www.hindawi.com

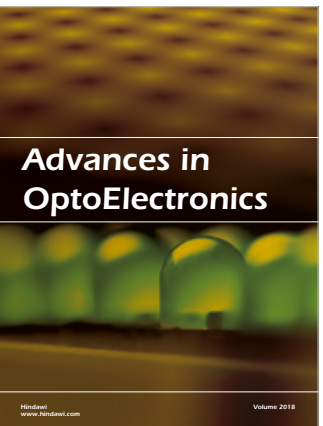

\section{Rotcting Machinery}
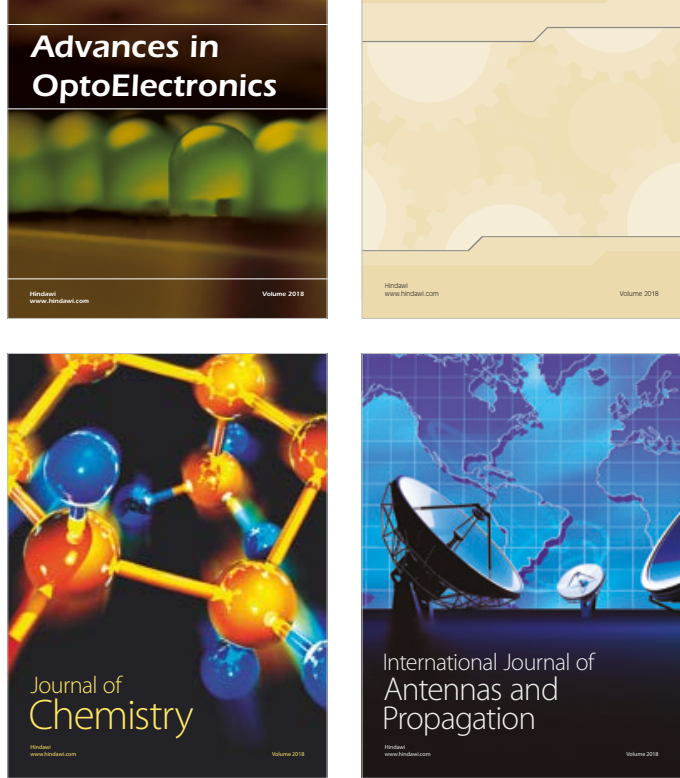

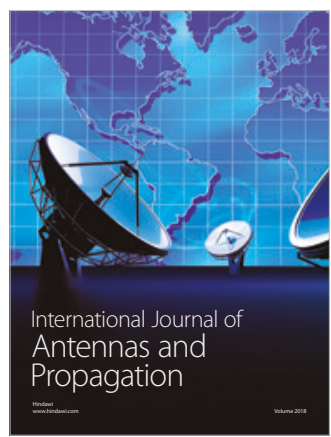

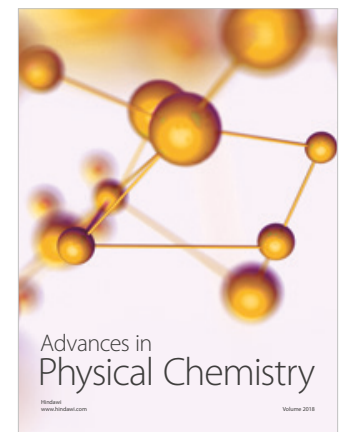

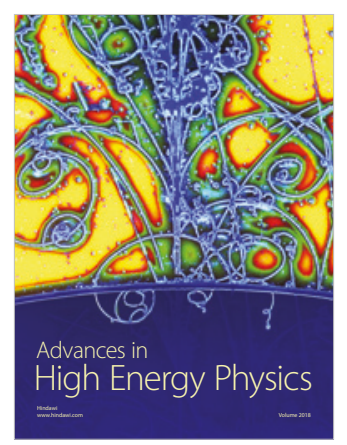

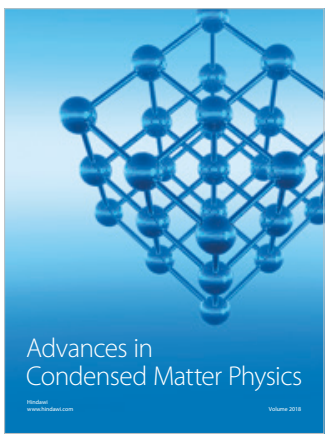

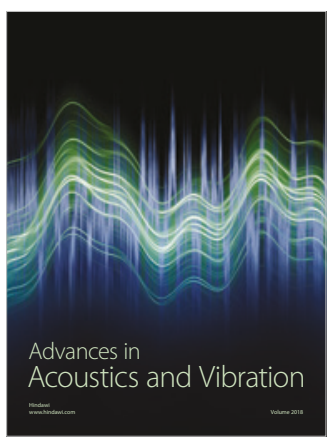

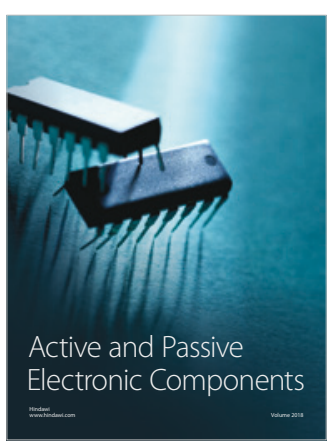
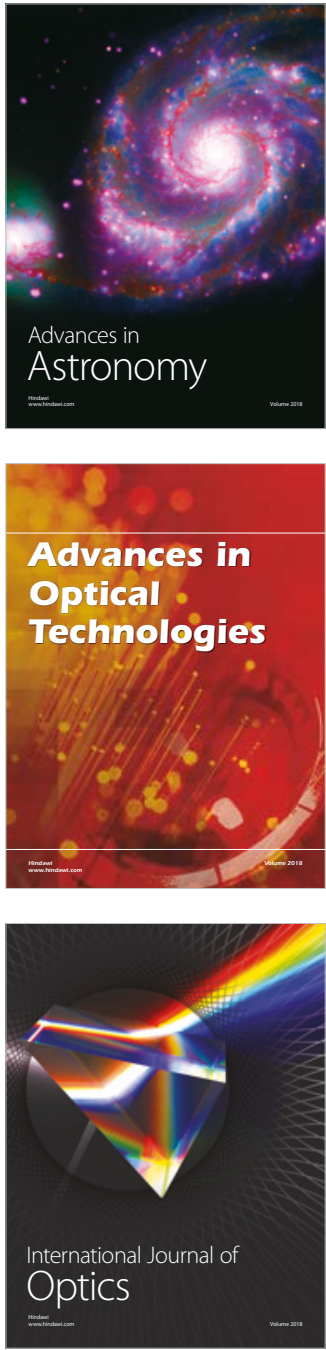